\title{
Image Reconstruction Based on Combination of Inverse Scattering Technique and Total Variation Regularization Method
}

\author{
Nor Haizan Jamali ${ }^{1}$, Kismet Anak Hong Ping ${ }^{{ }^{2}}$, Shafrida Sahrani ${ }^{3}$, \\ Dayang Azra Awang Mat ${ }^{4}$, Mohamad Hamiruce Marhaban ${ }^{5}$, Mohd Iqbal Saripan ${ }^{6}$, \\ Toshifumi Moriyama ${ }^{7}$, Takashi Takenaka ${ }^{8}$ \\ 1,2,3,4 Applied Electromagnetic Research Group, Department of Electrical and Electronic Engineering \\ Faculty of Engineering, Universiti Malaysia Sarawak, 94300 Kota Samarahan, Sarawak, Malaysia \\ ${ }^{5,6}$ Department of Electrical and Electronic Engineering, Universiti Putra Malaysia, \\ 43400 UPM Serdang, Selangor, Malaysia \\ ${ }^{7,8}$ Department of Electrical and Electronic Engineering, Faculty of Engineering, \\ Nagasaki University 1-14 Bunkyo-machi, Nagasaki 852-8521, Japan \\ ${ }^{*}$ Corresponding author, e-mail: hpkismet@unimas.my
}

\begin{abstract}
The Forward-Backward Time-Stepping (FBTS) had proven its potential to reconstruct images of buried objects in inhomogeneous medium with useful quantitative information about its size, shape, and locality. The Total Variation regularization method was incorporated with the FBTS algorithm to deal with the ill-posedness or ill-conditionedness of the inverse problem. The effectiveness of the proposed technique is confirmed by numerical simulations. The numerical method was carried out on simple object detection through FBTS with and without TV regularization method. The detection and reconstruction of relative permittivity and conductivity of the simple object have shown an improvement as TV regularization method applied whereas it smoothed the vibrations of the images and gave a better estimation of the image's boundaries.
\end{abstract}

Keywords: inverse scattering, regularization, total variation regularization

Copyright $\odot 2017$ Institute of Advanced Engineering and Science. All rights reserved.

\section{Introduction}

Microwave imaging is a technique used to sense an object or objects by means of analyzing microwave signals. Extensive researches have been conducted on microwave imaging and it had proven its potential utility and effectiveness for wide range of applications such as in medical imaging, remote sensing of earth, and non-destructive testing and evaluation. For example, in remote sensing, Synthetic Aperture Radar (SAR) is a form of radar system that has the capability to produce a high resolution imagery of bio and geophysical parameter of the Earth's surface [1-2]. In Non-Destructive Testing and Evaluation (NDT \& E), high frequency electromagnetic energy is utilized to determine material characterization and its structural integrity. Pulsed Eddy Current (PEC) is among the popular technique applied in NDT \& $E$ due to its effectiveness of quantifying defects in multilayer structures. Some applications involving non-destructive testing and evaluation are identifying hidden defect characterization in some complex structures [3], and defect detection of riveted structures of aging aircraft [4]. Microwave imaging applications for medical purposes had pulled a great attention and continuously investigated by many researchers. Medical imaging is the visualization of a biological system for clinical diagnosis and medical intervention proposes. Medical imaging modalities examples are X-ray, Ultrasound, Computerized Tomography (CT), Positron Emission Tomography (PET) and Magnetic Resonance Imaging (MRI). In microwave tomography, transmitting antennas are used to illuminate the biological part in microwave region and the scattered field data are then collected by receiving antennas. The scattered field information will be processed and analysed to reconstruct the dielectric properties of the biological part. The dielectric properties concern are the relative permittivity, $\varepsilon_{r}$, the capacitance of a unit volume of matter and conductivity, $\sigma$, the conductivity of a unit volume of matter. Microwave tomography 